\title{
Development of a Machine Translation System for Date Referencing in Yoruba Language
}

\author{
Durodola Folasade Olayinka \\ Department of Mechatronics Engineering, \\ Federal University of Agriculture, Abeokuta.
}

\author{
Eludiora Safiriyu ljiyemi \\ Computer Science and Engineering Department, \\ Obafemi Awolowo University, Ile-Ife.
}

\begin{abstract}
This study collected data on various format of writing date in English language and investigated the process underlying their translation into Yoruba language text. It formulates a date referencing model for the translation process, implements the model and evaluates the system with a view to developing a system that translate date to Yoruba language. The Yoruba language date translation process was achieved by identifying and extracting a date phrase in English language using regular expressions, the extracted date phrase is then converted to the British pattern of date referencing which is the $\mathrm{DD} / \mathrm{MM} / \mathrm{YYYY}$. It is this pattern of date referencing that is then translated to Yoruba language textual format. A ContextFree Grammar (CFG) that capture the various patterns of date referencing in English Language was specified. The CFG to capture the English language date phrase was modeled using the Push-Down Automata (PDA) and the software for the translation of the English language date phrase to the corresponding Yoruba language phrase was implemented with the Python programming language with the PyQt5 Graphical User Interface (GUI) module. The evaluation of the system was carried out using the Mean Opinion Score (MOS). The MOS was done using 45 questionnaires administered to some Linguistics students and staff of the News department, Orisun FM, (Radio station) Oke Itase, Ile-Ife, Osun state, Nigeria, to elicit their knowledge of the translation of the selected date phrases. The responses were compared with the output of the system. A desktop application for the translation process was deployed for windows operating system. The results of the system's evaluation showed that the application was able to translate the English language date phrases to Yoruba language text and produce 100 percent accuracy relative to a Yoruba language Expert translation.
\end{abstract}

The result of the respondents to the questionnaires shows that most of the respondents are not familiar with the orthography of the Yoruba language, which means that a large percentage of Yoruba language native speakers cannot tone mark their responses correctly. Another observation was that most of the respondents could not translate the year numbers to Yoruba language as they are not familiar with translation of large numbers. In conclusion, this study has shown that date referencing translation process can be computationally analyzed using the modern knowledge of computing.

\section{Keywords}

Keywords: Translation process, Date, Calendar, number, Yoruba Language, Year, knowledge

\section{INTRODUCTION}

No event no history. History takes account of events. In taking the accounts of events the dates are referenced. Date plays important roles in human activities. The language and culture of many African countries use some events like festival or some cultural activities to fix the date of events. For example, Olojo festival in Ile-Ife, new yam festival in Igbo land. This study experiments the translation of date phrase from English language to Yoruba language in order to integrate into the body of English to Yoruba machine translation system. Some researchers have worked on phrases and sentences machine translation systems for the English and Yoruba languages but their studies did not address date referencing.

A web-based Yoruba language translation system was developed by [1] to address Yoruba language endangerment particularly on numeral. The study enumerated how number in English language can be translated to Yoruba language. User can easily translate any number from English language to Yoruba language. The date pattern is different from numeral pattern. The numeral translation system provided useful information for date referencing translation system.

\section{LITERATURE REVIEW}

Machine Translation is the process of using the computer system to translate text from a source language to a target language with the aim of retaining the meaning of the source language text in the target language text [2]. There exist two levels of machine translation: Meta phrase and paraphrase [3]. The meta phrase is a word to word translation while the paraphrase has more to do with meaning of the original text being translated. The paraphrase level has three approaches;

i. Statistical machine translation.

ii. Rule - based machine translation.

iii. Hybrid machine translation.

Statistical machine translation is a system that takes the corpora from both the source and target language using a generic model. It proceed to generate rules for the translation of the languages involved by finding the probability of a word translating to another [4]. In other words, computing power is used in building models for translating from a source language to the target language. Dataset is used for the training of the model using algorithm to select the most occurring words or phrases. Bilingual corpus such as bilingual dictionary and glossary are necessary for the database, hence it is considered expensive and resources intensive.

Rule-based machine translation is also known as knowledge based machine translation. Its approach is based on linguistics information on both the source and the target language [5]. The author applies syntactic and semantic rules on both languages [6].The study uses bilingual dictionaries of both languages. The limitation to the approach is the exceptions in some grammars. The RBMT is of three types: 
1. Direct Systems or Dictionary Based Machine Translation Systems.

2. Transfer RBMT Systems of Transfer Based Machine Translation Systems.

3. Interlingual RBMT Systems or Interlingua.

The direct systems approach uses dictionaries of both languages. The input text is broken into tokens or words and the words equivalent is used to build the lexicon.

In the transfer RBMT systems approach, the syntactic or semantic structure of source language is transferred into the syntactic or semantic structure of the target language.

Lastly the interlingual RBMT systems is considered as the third generation machine translation. Here, the source language is converted into an intermediate language which is independent of source and target languages. Analysis and synthesis are all required in this mode of translation and because it does not depend on the source or target language, this system has much relevance in multilingual machine translation [2].

The Hybrid machine translation is an approach of machine translation where more than one machine translation are in the translation process. The idea is conceived from the fact that some machine translation approaches has one setback or failure in one form or the other. In other to make up for this failures, a hybrid approach is used. HMT is the combination of both RBMT and SMT. It is an area undergoing development, so it is expected that many system will evolve into hybrid platforms. There are two types:

Rule base that uses statistical translation for post processing and cleanup, and statistical systems that are guided by rule based engines.

\subsection{Calendar}

Calendar is defined as time measurement which shows how months and years are reckoned. The calendar is a system that gives an identity to each day [7]. From time in memorial, every tribe and culture has a means of reckoning time and date, whether by mental computation or otherwise, a calendar is birth in the process [8].

Calendars are social constructs which have their roots in astronomy [9]. The astronomic basis of the calendar are the day, the month and the year cycle [10]. The study of each of these cycles birth the calendar. Calendar is mankind's attempt to choreograph the eternal dance of the Sun, Moon and Earth [11].

There are three main types of calendars; the lunar calendar, the luni-solar calendar and the solar calendar [12]. The lunar calendar is based on the Moon. A lunar calendar year is 12 months long and the month is the time interval in which the phases of the Moon repeat, i.e. from one full moon to the next, and averages 29.53 days [13]. Therefore, a lunar calendar year is usually 354.37 days long. Because Earth takes slightly longer than 365 days to revolve completely around the Sun, a lunar calendar soon gets out of phase with the seasons. Examples of the lunar calendar are the Gezer calendar, the Nepal sambat calendar, the Islamic calendar, Assyrian calendar, etc. Most lunar calendars have phased out over the centuries, the main exception is the Islamic calendar, which is used in Islamic countries [14].

The second type of calendar is the luni-solar calendar which is 12 months long, with a thirteenth month which is usually inserted every few years to keep the calendar in phase with the seasons. The luni-solar calendar uses a combination of the lunar and solar activities to set the date. Example of the lunisolar calendar are Attic calendar used in the classical Athens and was introduced around 6th century BC, old Persian calendar which was introduced around 4th century BC and used in the Persian Empire [15].

The Genesis calendar, Ptolemaic calendar, the Hebrew calendar and the Chinese calendar are other examples. Most of these have gone into extinction except for the Hebrew calendar, which is used by Hebrew nations for the Jewish religious activities, and the Chinese calendar, which is used extensively in Eastern Asia [16].

The third type of calendar is the solar calendar, which is based on the length of the year, particularly the yearly solar cycle [12]. Examples of the solar calendar are Roman republican calendar which was in used $713 \mathrm{BC}$ in the Roman Empire, Julian calendar which was used around $45 \mathrm{BC}$ in the western world, and the Coptic calendar which was introduced in Egypt in the first century. The

Gregorian calendar is of this type; however, it evolved from the ancient Roman calendar, which passed through the stage of being a luni-solar calendar. For the purpose of this work, the focus is on the Gregorian calendar.

\subsection{Date}

Date is defined as a numbered day in a month, often given with a combination of the name of the day, month and year [17]. From another perspective, date is defined as a particular month, day and year at which some event happened, an inscription on a writing, coin, etc. that shows the time of writing, casting, delivery, etc. it can also be used to express the duration of an activity [18]. From all of these definitions and many more ways through which the term date (in this context) could be understood and used, we would be adopting the first definition which considered date as a numbered day in a month, often given with the combination of the name of the day, month and year. In order words, date is the identifier of each day that breaks in relation to the month and the year, as outlined by a specific calendar.

The study has been organized into sections. Section 2 deals with data collection. Section 3 deals with the methodology of the study in which machine translation for date referencing is developed. Results, discussion and conclusion are discussed in Section 4, 5 and 6 respectively.

\section{METHODOLOGY}

In this section, the materials and methods used for the developed system are discussed. The design principles or approaches are explained.

\subsection{Data Collection}

In order to be able to implement a system for translating a date phrase, there's a need to collect data from linguistic and study its translation process. Data were collected on date phrase in English language and the date phrase was translated into Yoruba taking their context in the English text into consideration. This section contains the process involved in the collection of data (date referencing patterns in English phrases) and how they were translated to the corresponding Yoruba text.

Data collection was done by consulting books, online text and newspapers. Also, processed food (such as drinks, biscuit, pastas, and baby cereals), drugs etc. and bilingual dictionary 
were consulted. The newspapers consulted are Nigeria newspapers such as sun news, the punch newspaper, the tribune, vanguard newspaper. Also some online articles which are properly dated were sourced. It was discovered during the course of research that there are various ways or pattern of referencing the date. Table 1 are some of the common date patterns currently in use.

1. YYYY/MM/DD: this is a style where the year is written first, and denoted with four digit followed by the month which is depicted with two digit, and lastly by the numeral representation of the day, also in two digit. This is the style recognized according to the ISO8601 standard of writing date. Example is 2017/12/25.

2. DD/MM/YYYY: this is a style where the day is represented first with two digit, followed by the month which is also represented by two digit or by the name of the month, then lastly the year is represented with four digits. This is the British pattern of writing date which has generally been accepted and adopted in Nigeria. Example is 01/10/2017. Another example is 01- Jan, 2098.

3. MM/DD/YYYY: In this style, the month is denoted first with either two digit or the name of the month, then followed by the day which is also represented by two digit, and lastly by the year which is represented by four digit. Example is 29/06/2000.

4. DD (order) MM, YYYY: In this style, the day numeral is written first and it carries an order of the numeral, e.g. 1st, 2nd, 3rd, 4th. Next is the name of the month which can be in full or in part, e.g. Oct. or October. And lastly, the year is depicted with four digit. There is no separator between the day and the month, but a comma comes immediately after the name of the month. Examples are "3rd July, 1887", "1st Oct., 2019" etc.

5. MM DD (order), YYYY: This pattern first references the month by its name and then reference the day number with its order. The month can be written in part or in full. A comma comes immediately after the order of the day number. Lastly, the year is depicted by four digits. Examples are "Sept. 5th, 2089", "January 18th, 2000."

Table 1: Patterns of Date Referencing

\begin{tabular}{|c|c|}
\hline Date Pattern & Example \\
\hline $\mathrm{dd} / \mathrm{mm} /$ yyyy & $22 / 01 / 2012$ \\
\hline dd-mm-yyyy & $23-09-2089$ \\
\hline dd.mm.yyyy & 30.09 .1987 \\
\hline mm/dd/yyyy & $06 / 30 / 2098$ \\
\hline yyyy.mm.dd & 1987.02 .30 \\
\hline dd(order)mm, yyyy & 3rd July. 1887 \\
\hline mm dd(order), yyyy & September 18th, 2099 \\
\hline mm. dd(order), yyyy & Oct. 23rd, 2089 \\
\hline dd(order) mm., yyyy & 3rd Oct., 2076 \\
\hline
\end{tabular}

Table 2: System Library

\begin{tabular}{|c|c|c|c|}
\hline Input & Day & Month & Year \\
\hline 15th May, 2001 & 15 & May & 2001 \\
\hline July 4th, 2012 & 4 & July & 2012 \\
\hline $2099-06-12$ & 12 & 6 & 2099 \\
\hline $23 / 05 / 1980$ & 23 & 05 & 1980 \\
\hline $06 / 30 / 1989$ & 30 & 06 & 1989 \\
\hline 15th Sept., 2011 & 15 & 09 & 2011 \\
\hline Oct. 17th, 2200 & 17 & 10 & 2200 \\
\hline 1980-05-23 & 23 & 05 & 1980 \\
\hline $2009 / 01 / 02$ & 02 & 01 & 2009 \\
\hline
\end{tabular}

\subsection{Preprocessing of Data}

Preprocessing or normalization of data eliminates numerical difficulties or condition where the values of the months and the days can be mixed up. For example, the date 08/06/2000 can be referenced as 8th June, 2000 and also as 6th August, 2000. The normalization process occur in the system library where the data goes through a conversion process. The conversion process entails identification and capturing any of the existing date format patterns as explained in Table 1, with the aids of regular expressions. Next the identified date is converted to the DD/MM/YYYY format which is then saved in the system library. The Table 2 shows how the processed dates are saved in the system library

\section{MODEL FORMULATION AND DESIGN}

The translation model is formulated using English grammar. A grammar is a set of rule guiding the formation of a language $\sum^{*}$ for some alphabet $\sum$. In order to capture the different patterns of referencing date and convert to a particular pattern, a machine that has memory is needed in capturing numbers and words used in the context of date for proper translation. Therefore, to capture the various date formats, formulation was done using Context Free Grammar (CFG). The machine to process this kind of grammar is a Push Down Automata. The Push-Down Automata is a machine that combines Finites Automata (FA) with a stack memory. Each date format are converted to the DD/MM/YYYY format in the stack and popped out for validation and translation. The grammar production rule For Date in English is as follows:

$$
\mathrm{G}::=<\mathrm{D} ; \mathrm{T} ; \mathrm{P} ; \mathrm{S}>
$$

$\mathrm{G}$ implies the grammar,

D implies the non-terminal symbols,

$\mathrm{T}$ implies the terminal symbols and

$\mathrm{P}$ implies the production rule.

$\mathrm{S}$ is the start symbol.

$\mathrm{D}=$ Date, DP, MP, YP D, O, SF, $\mathrm{MW}_{\mathrm{s}}, \mathrm{MW}_{\mathrm{L}}$

$\mathrm{T}=$ list of $\mathrm{D}$, List of $\mathrm{S}$, List of SF, List of $\mathrm{MW}_{\mathrm{s}}$, List of $\mathrm{MW}_{\mathrm{L}}$

$\mathrm{S}=$ Date

Date $\langle\mathrm{DP}\rangle\langle\mathrm{O}\rangle\langle\mathrm{MP}\rangle\langle\mathrm{O}\rangle\langle\mathrm{Y} \mathrm{P}\rangle$ (2)

Date $\langle\mathrm{MP}\rangle\langle\mathrm{O}\rangle\langle\mathrm{DP}\rangle\langle\mathrm{O}\rangle\langle\mathrm{YP}\rangle(3)$ 


$$
\begin{aligned}
& \text { Date }\langle\text { Y P }\rangle\langle\mathrm{O}\rangle\langle\mathrm{MP}\rangle\langle\mathrm{O}\rangle\langle\mathrm{DP}\rangle \text { (4) } \\
& \mathrm{DP}\langle\mathrm{D}\rangle\langle\mathrm{D}\rangle|<\mathrm{D}\rangle\langle\mathrm{D}\rangle\langle\mathrm{SF}\rangle \\
& \mathrm{MP}<\mathrm{D}\rangle\langle\mathrm{D}\rangle\left|<\mathrm{MW}_{\mathrm{S}}\right\rangle\left|<\mathrm{MW}_{\mathrm{L}}\right\rangle \text { (6) } \\
& \mathrm{YP}\langle\mathrm{D}\rangle\langle\mathrm{D}\rangle\langle\mathrm{D}\rangle\langle\mathrm{D}\rangle \\
& \mathrm{D}\langle 0 ; 1 ; 2 ; 3 ; 4 ; 5 ; 6 ; 7 ; 8 ; 9> \\
& \mathrm{O}<., \ldots \text {, / }>
\end{aligned}
$$

\section{SOFTWARE DESIGN AND IMPLEMENTATION}

This section discusses software design and implementation.

\subsection{Software Design}

Software design which includes system flowcharts is being reported here. The flowcharts described the procedures for the software development. The flowcharts explain step by step software coding. Tagged words which are the names of the months had been stored in the database. When a text is typed, the system checks if there is a valid date phrase in it, if it yes the date phrase is extracted putting into consideration the rules of the Gregorian calendar and the leap year, if the date is valid, textual numbers in the date phrase are converted to Arabic digit representation. The system sends the date to the parser module otherwise the translation process is terminated. The re-write rules designed for the two languages are used to determine the correctness of the dates. The system parses the date if it agrees with the rules, otherwise, the translation is terminated. The system generates value errors when it cannot translate the date. New date can be typed when the reset button is pressed, otherwise, the application can be closed. The translation processes involve all the steps mentioned in the flowcharts shown in Figure 2.

\section{SYSTEM IMPLEMENTATION}

In this section, software implementation is reported. Natural language tool-kit (NLTK), Python, PyQt4, and file-based custom database are the tools used for the software implementation. The NLTK (is a version of python programming language) was used for the sentence parsing. The NLTK was used to determine the correctness of the rewrite rules designed. The phrase structure grammar model was used for the re-write rules. The PyQt4 (a version of Python) was used for the implementation of the graphical user interface (GUI). The GUI has two planes. The first plane is where the English date text can be typed. The second plane displayed the translated Yoruba language date.

\section{System Output}

The user can type the source language date phrase through the first plane. The user clicks the translate button. If some of the words are not in the library, the system generates errors. Though only the administrator can add the new words to the various lexemes' databases. Figures 3, 4 and 5 are the sample outputs of the date referencing translation system. Figure 3 is the date phrase with the pattern DD/MM/YYYY. Figure 4 is a date phrase with the pattern mm, dd (order), yyyy. Figure 5 is a date phrase with the pattern $\mathrm{mm}$, dd (order), yyyy.

\section{SYSTEM PERFORMANCE EVALUATION}

The evaluation process involves the questionnaire design, detailing Expert and Experimental subject respondents (ESRs) profiles, and questionnaire administration.

\subsection{Questionnaire Design}

The questionnaire was developed with the aim of carrying out syntax, semantic and orthographic analysis on the responses received. In order to perform the evaluation, 50 questionnaire were administered and 45 was returned. The questionnaire contained 14 date phrases: 3 date phrase to cover the $\mathrm{DD} / \mathrm{MM} / \mathrm{YYYY}$ format, 3 date phrase to cover the MM/DD/YYYY format, another 3 phrase to cover the YYYY/MM/DD format, 2 date phrase to cover the DD(st, nd, th,

rd) month, YYYY format, and the last 2 date phrase to cover the month dd(st, nd, rd, th), YYYY format.

\subsection{Expert and Experimental Subject Respondents' (ESRs) Profiles}

The profile of the ESRs and the Expert were captured in the questionnaire. The returned questionnaire provided the needed information such as age range, sex, states, educational level, and knowledge of Yoruba orthography about each

ESR. The ESRs are postgraduate, graduate and undergraduate students with ages range between 20 and 50 years. Majority are above 30 years of age. Their Yoruba orthography knowledge range between adequate and excellent. The

ESRs and Human Expert are literate Yoruba speakers. The human expert (HE) has a $\mathrm{PhD}$ in Yoruba language, and is a trained translator. $\mathrm{He}$ teaches Yoruba language and linguistics. He also anchors a Yoruba educating program on a radio station. The human expert translations were used to moderate the score of machine and ESRs translations during the system performance evaluation.

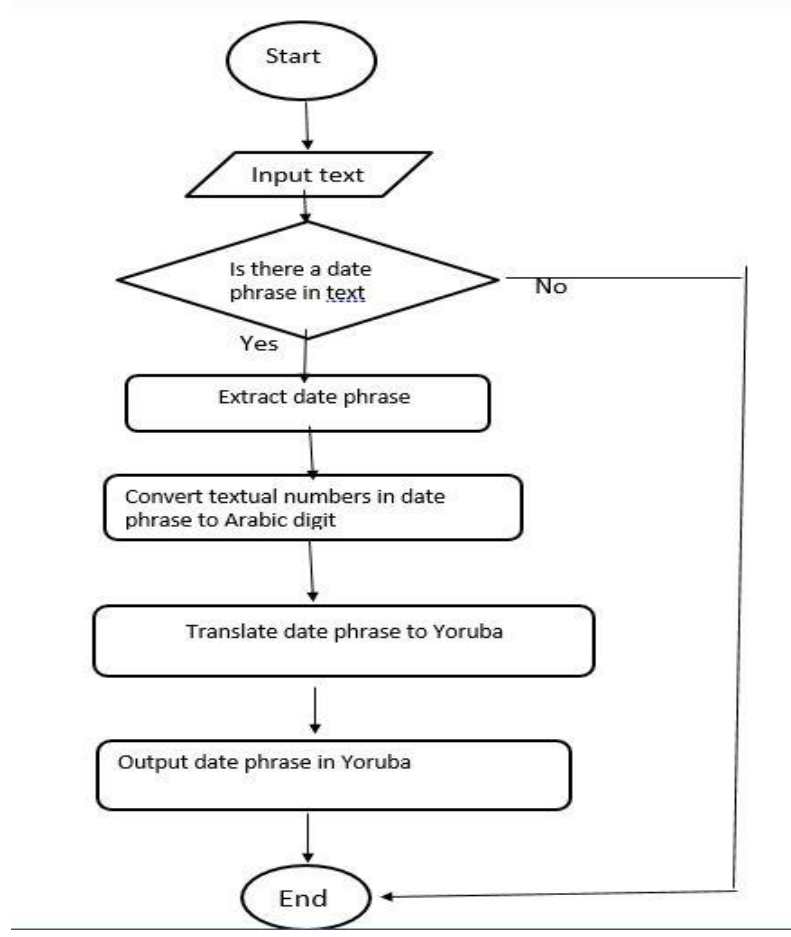

Figure 2: System Flowchart 


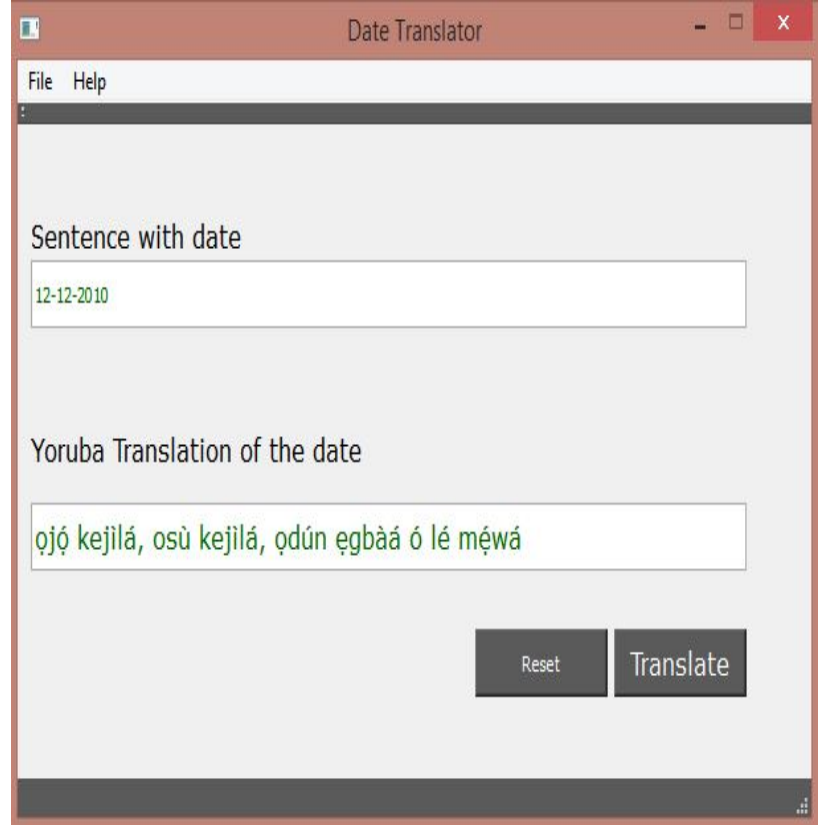

Figure 3: The date phrase with the pattern DD/MM/YYYY

\subsection{Questionnaire Administration}

The instrument comprises fourteen (14) date phrase administered to fifty (50) (ESRs). The distribution was done within the Obafemi Awolowo University, Ile-Ife, Nigeria. This environment was suitable because literate Yoruba speakers are needed to do the translations. The questionnaires were distributed among Yoruba ethnic group only. Forty-five ESRs returned the questionnaires. The evaluation was carried out on 14 translated date phrase for forty-five ESRs, excluding the machine translated sentences.

\subsection{System Evaluation results}

We evaluated the system by considering some different patterns/formats of date referencing. We considered the ESRs and the machine translated sentences. The expert translations are used to control the choice of words between the ESRs and the machine translated date phrase. The three criteria used for the evaluation are word semantic accuracy, word orthography, and sentence syntax correctness. Word orthography accuracy means that a word in a sentence is properly tone marked and under-dotted. For example, a word Odonn has two syllables, $Q$ has one syllable with mid-tone which also has an under dot and donn has one syllable with a high tone on $\mathrm{u}$. We evaluated each word using this approach and we scored the ESRs and the machine based on their ability to identify these syllables. The word semantic accuracy was evaluated by considering the meaning of each word within the sentence. All these and some other Yoruba writing styles were considered during the evaluation. The ESRs and the machine translated phrases were scored. The phrase syntax correctness was evaluated based on the phrase structure of the target language (TL). We considered the position of each word in a phrase. A wellpositioned word in a phrase attracts good score.

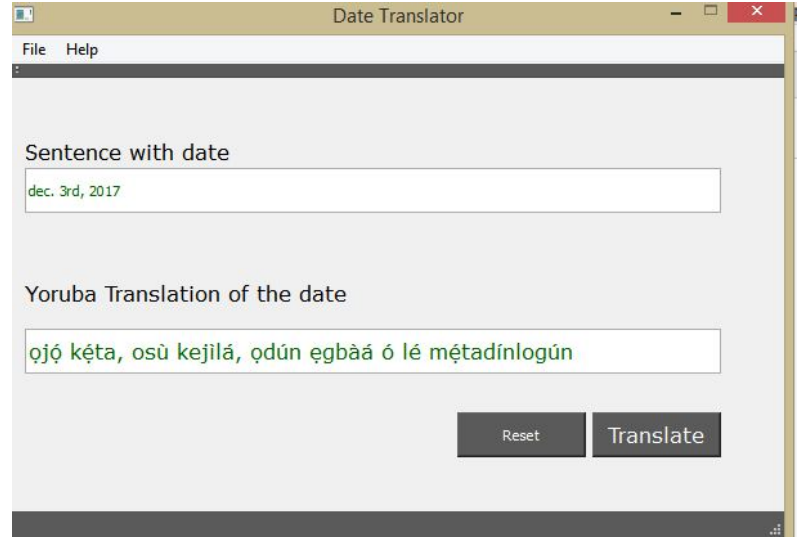

Figure 4: The date phrase with the pattern mm dd(order) yyyy

\begin{tabular}{|l|l|}
\hline File Help & Date Translator \\
\hline Sentence with date \\
\hline December 12tht, 2010 \\
\hline Yoruba Translation of the date \\
\hline ọjọ́ kejilá, osù kejilá, ọdún ẹgbàá ó lé mẹ́wá \\
\hline \\
\hline
\end{tabular}

Figure 5: The date phrase with the pattern $\mathrm{mm}$ dd (order), yyyy

\section{DISCUSSION}

This section discusses the results of the evaluation based on three criteria: orthography, syntax and semantic accuracies. Yoruba is a tonal language, the word meanings rely on the tone marks. The tone marks resolve the possible ambiguity that do occur in some words. The question is, does the ERS knows the rules that guide tone marking of words. Information about how each ESR tone marked the word were collated and use for the award of marks. Syntax accuracy simply means how does ESR understand the rules under lining the sentence or phrase syntax structure. Yoruba language is head first unlike English that is head last. Semantic accuracy asked the question, does the response of ESR provides adequate meaning to the sentence/phrase. It is should be noted that we compared system and experimental subject respondents (ESRs) performances with the Expert. On the graphs, percentage of correct responses is meant for the ESRs. The average Expert scores is always 100 percent.

\subsection{Word orthography accuracy}

Figure 6 shows the results of Word orthography accuracy of date phrase 1 to 14 . It was observed that the developed system's scores are higher than that of the experimental subject respondents (ESRs). The scores of the developed system are equal to that of the Expert except for one instance, where the score was about 90 percent as it is shown in Figure 6. Also there is no instances where the score of ERSs' average score is equal to that of the developed system. The respondents' average score is 35 percent, while the developed system average score is 99.1 percent. However, it was noticed 
that more than two third of respondents did not tone mark the words. This was responsible for the performance recorded by these ESRs. This reduces the ESRs average scores. The results present the extent of familiarity of the respondents to orthography of the Yoruba language.

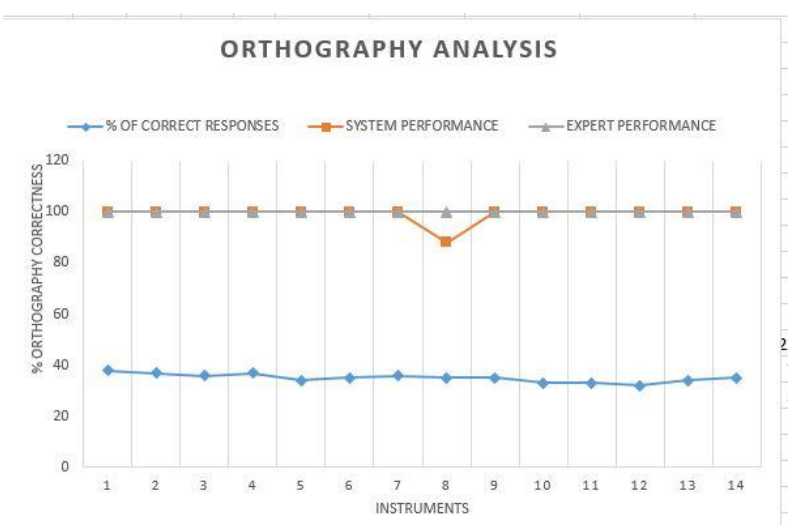

Figure 6: Word orthography accuracy

The major priority here is on the use of tonal marks and under dots where necessary. The results show that only three (3) of the respondent have a very good knowledge of Yoruba language orthography. Another seven (7) respondents have an average understanding while thirty-five (35) respondents performed badly.

\subsection{Word syntax accuracy}

The analysis carried out to determine familiarity of the respondents on the syntax of date phrase in Yoruba language. The analysis indicates that most of the respondents were not familiar with the syntax of date phrase in Yoruba language as only seven (7) respondents were able to arrange the date phrase correctly. Figure 7 shows the results of syntax accuracy of different date phrases

1 to 14 .

\section{SYNTAX ANALYSIS}

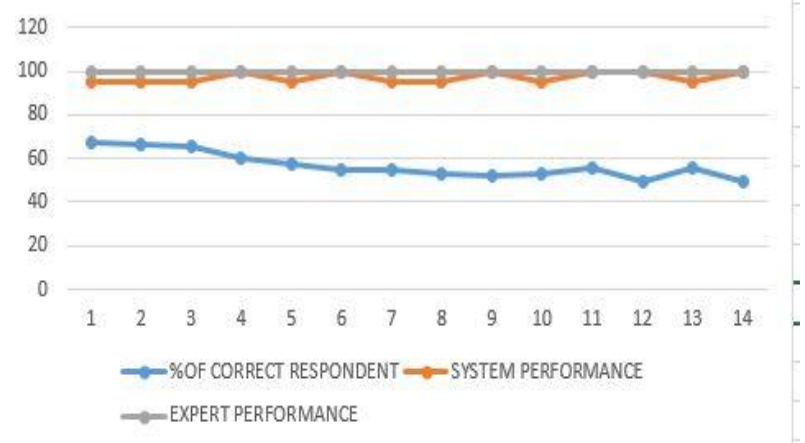

Figure 7: sentence syntax correctness

It was observed that the developed system scores higher than that of the experimental subject respondents (ESRs), its scores almost equal to that of the Expert except for few instances where the scores are below that of the Expert. There are no instances where the ESRs scores are equal to that of the Expert as it is shown in Figure 7. The respondents' average score is 56.9 percent, the developed system average score is 97 percent and the Expert average score is 100 percent.

\subsection{Word semantic accuracy}

The analysis carried out to determine the correct number of respondents who understands the correct meaning of the date phrase. This is done in order to validate their familiarity with the numbers and words used in the date phrase and how they are used in the context of date. The scores of the machine translator are equal to that of the Expert except for one instance as it is shown in Figure 8.

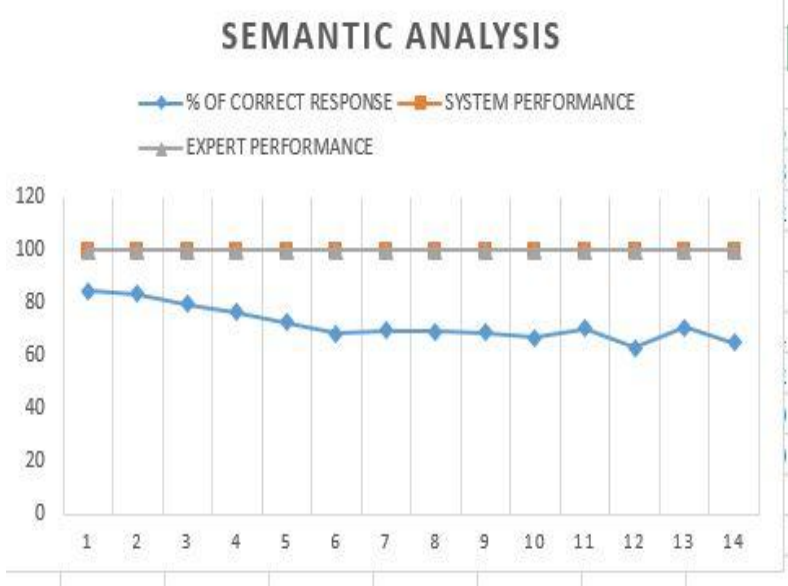

Figure 8: Word Semantic Analysis

Also there is no instances where the score of ESRs' average score is equal to that of the Expert. The respondents' average score is 64.9 percent, the machine translator average is 100 percent. The analysis done in Figure 6, 7 and 8 clearly show the comparison between the respondents and the developed system. This validates that the developed system performs relatively well compare to the respondents in terms of accuracy.

\section{CONCLUSION}

The Authors studied and analyzed the English date phrases with focus on extracting the knowledge needed and the conditions required for its translation into standard Yoruba. It has been pointed out and discussed that there are various patterns of date referencing amongst nations of the western world. Authors discussed the phrasal context on the dates in English language and how they are translated to the corresponding Yoruba text. Also, presented a grammar capturing the English date phrase and a model for the recognition. The processes involved in the translation has been discussed. The results of the study have been presented and discussed with a view to provide a key to the proper date referencing in standard Yoruba language. In this study, Authors found out that:

1. There are various patterns of date referencing with peculiarities in the date compositions and arrangements, how they are translated from English phrasal context and the linguistic rules involved in their pronunciation.

2. The software developed was able to give $100 \%$ accuracy on tested data.

Future work. In future, Authors will develop Yoruba language date referencing patterns that can be used in schools and public places like Gregorian calendar.

\section{ACKNOWLEDGEMENTS}

Special thanks to everyone who have contributed towards development of the system.. 


\section{REFERENCES}

[1] O.A. Agbeyangi, and S.I. Eludiora and O.A. Popoola, Web-Based Yoruba Numeral Translation System, IAES International Journal of Artificial Intelligence (IJ-AI) 5 $\begin{array}{lll}\text { (4) (2016) } 127\{134 & \text { (2016). doi:10.1016/S0031- }\end{array}$ 8914(53)80099-6.

[2] S. Tripathi, J. K. Sarkhel, Approaches to Machine Translation, Journal of Scientific and Industrial Research 57 (4) (2010) $388\{393$ (2010).

[3] Y. Al-Onaizan, J. Curin, M. Jahr, K. Knight, J. La_erty, D. Melamed, F.-J. Och, D. Purdy, N. A. Smith, D. Yarowsky, Statistical machine translation, in: Final Report, JHU Summer Workshop, Vol. 30, 1999 (1999).

[4] W. Weaver, Translation, Machine translation of languages 14 (1955) $15\{23$ (1955).

[5] S. I. Eludiora, Development of an English to Yoruba Machine Translation System, Unpublished PhD Thesis, Obafemi Awolowo University, Ile-Ife.(2014).

[6] M. Osborne, Machine Translation History and RuleBased Systems, School of Informatics, University of Edinburgh 16 (6) (2012) 109\{129 (2012).

[7] McGraw-Hill, McGraw-Hill concise encyclopedia of physics, McGraw-Hill Companies, New York, United States, 2005 (2005).

[8] V. G. Chivaura, Understanding Nanotechnology from an African Worldview, International Open and Distance Learning Journal 1 (1) (2015) 59\{63 (2015).

[9] A. Longsta_, Calendars from Around the World, Greenwich: National Maritime Museum 25 (55) (2005) $42\{49$ (2005).

[10] C. Handelman, Calendar: Humanity's Epic Struggle to Determine a True and Accurate Year, Comitatus: A
Journal of Medieval and Renaissance Studies 30 (1) (1999).

[11] W. Gleissberg, Astronomical Cycles: An Unexpected Anomaly in the Annual Distribution of the Maxima of the 11-year Sunspot Cycle, Taylor and Francis, 1975 (1975).

[12] D. H. Clark, J. H. Parkinson, F. R. Stephenson, An Astronomical ReAppraisal of the Star of Bethlehem-A Nova in 5 BC, Quarterly Journal of the Royal Astronomical Society 18 (2) (1977) $443\{449$ (1977).

[13] M. R. Salzman, the Representation of April in the Calendar of 354, American Journal of Archaeology 6 (5) (1984) $43\{50$ (1984).

[14] S. I. Eludiora, A. F. Agboola, O. A. Odejobi, T. V. Abijo, Indigeneous Calendar System for Time and Date Reckoning: Computational Analysis and Software Development in LINGUISTICS AND THE GLOCALISATION OF AFRICAN LANGUAGES FOR SUSTAINABLE DEVELOPMENT,

[15] UNIVERSAL AKADA BOOKS NIGERIA LIMITED, IBADAN, 2013 (2013).

[16] H. Aslaksen, The Mathematics of the Chinese Calendar,

[17] http://www.xirugu.com/CHI500/Dates, Time/Chinesecalender.pdf, Accessed on 19th december, 2017 (2003).

[18] J. Murray, Oxford English Dictionary, Oxford University Press, Oxford, United Kingdom, 2008 (2008).

[19] D. Macmillan, Thesaurus: Free English Dictionary Online, :http://www.macmillandictionary.com (accesssed on 4th july,2017) (2016).

[20] W. M. O'Neil, Time and the Calendars, Manchester University Press, 1976 (1976). 